\title{
Swift and Supergiant Fast X-ray Transients: a novel monitoring approach
}

\section{P. Romano ${ }^{*}{ }^{a}$ L. Sidoli, ${ }^{b}$ V. Mangano, ${ }^{a}$ J.A. Kennea, ${ }^{c}$ G. Cusumano,${ }^{a}$ S. Vercellone,${ }^{b a}$} H.A. Krimm, ${ }^{\text {def }}$ D.N. Burrows, ${ }^{c}$ N. Gehrels, ${ }^{f}$

a INAF, Istituto di Astrofisica Spaziale e Fisica Cosmica,

Via U. La Malfa 153, I-90146 Palermo, Italy

${ }^{b}$ INAF, Istituto di Astrofisica Spaziale e Fisica Cosmica,

Via E. Bassini 15, I-20133 Milano, Italy

${ }^{c}$ Department of Astronomy and Astrophysics, Pennsylvania State University, University Park, PA 16802, USA

${ }^{d}$ CRESST/Goddard Space Flight Center, Greenbelt, MD, USA

${ }^{e}$ Universities Space Research Association, Columbia, MD, USA

${ }^{f}$ NASA/Goddard Space Flight Center, Greenbelt, MD 20771, USA

E-mail: romanodifc.inaf.it

We describe our monitoring strategy which best exploits the sensitivity and flexibility of Swift to study the long-term behaviour of Supergiant Fast X-ray Transients (SFXTs). We present observations of the recent outbursts from two objects of this class. IGR J16479-4514, underwent an outburst on 2008 March 19, reaching a peak luminosity of about $6 \times 10^{37} \mathrm{erg} \mathrm{s}^{-1}(0.5-100 \mathrm{keV}$; at a distance of $4.9 \mathrm{kpc})$. We obtained a simultaneous broad-band spectrum $(0.3-100 \mathrm{keV})$, the first for the SFXT class, which is fit with a heavily absorbed (column density $5 \times 10^{22} \mathrm{~cm}^{-2}$ ) hard power-law with a high energy cut-off at about $7 \mathrm{keV}$. This spectrum shows properties similar to the ones of accreting pulsars, although no X-ray pulsations were found. IGR J11215-5952, one of the only two periodic SFXT known to date, was observed with Swift several times, first with an intense 23-day long monitoring campaign around the 2007 February 9 outburst; then with a 26day long monitoring around the unexpected July 24 outburst; finally with a deep exposure during the 2008 June 16 outburst. We present the whole dataset, which also includes observations which allowed us to firmly establish the outburst period at $P \sim 165$ days. Thanks to our combined observations common characteristics to this class of objects are emerging, i.e., outburst lengths well in excess of hours, often with a multiple peaked structure, dynamic range $\sim 3$ orders of magnitude, and periodicities are starting to be found.

7th INTEGRAL Workshop

September 8-11 2008

Copenhagen, Denmark

\footnotetext{
* Speaker.
} 


\section{Monitoring SFTXs with Swift}

Supergiant Fast X-ray Transients (SFXTs) are a new class of High Mass X-ray Binaries (HMXBs) discovered by INTEGRAL while monitoring the Galactic plane. They display outbursts which are significantly shorter than typical Be/X-ray binaries, peak luminosities in the order of a few $10^{36} \mathrm{erg} \mathrm{s}^{-1}$, and a quiescent level of $\sim 10^{32} \mathrm{erg} \mathrm{s}^{-1}$. Since their spectral properties are reminiscent of those of accreting pulsars, it is assumed that all the members of the new class are HMXBs hosting a neutron star, although the only three SFXTs with a measured pulse period are IGR J11215-5952 ( $P_{\text {spin }} \sim 187 \mathrm{~s}$, [24]), AX J1841.0-0536/IGR J18410-0535 ( $P_{\text {spin }} \sim 4.7 \mathrm{~s}$, [1] $)$, and IGR J18483-0311 $\left(P_{\text {spin }} \sim 21 \mathrm{~s}\right.$, [17] $)$. The actual mechanisms responsible for the observed short outbursts are still being debated, and the proposed explanations (see [21] for a review) involve either the structure of the wind from the supergiant companion [7, 25, 10] or gated mechanisms (see [2]).

As we reported in [11, 18], our Swift [5] monitoring of the outburst of the periodic SFXT IGR J11215-5952 in February 2007 represents the most complete and deep set of X-ray observations of an SFXT outburst. We discovered that the accretion phase during the bright outburst lasts longer than previously thought: days instead of hours, with only the brightest phase lasting less than one day.

Given the success of these Swift observations, we extended the investigation to a small although well-defined sample of SFXTs for which a clear periodicity in the outbursts recurrence had not been found yet-as, on the contrary, was the case for IGR J11215-5952-with a monitoring campaign that would span the length of at least a few periods, i.e. in the order of 100-300 days. The primary goal was to test whether our model [18] for the periodic SFXTs would also apply to some of the other members of the class. Within this model, based on the presence of a disk-like wind component from the supergiant donor, inclined with respect to the orbital plane, the outbursts are produced when the neutron star crosses the wind component along the orbit. This model implies a periodicity or a semi-periodicity in the outburst recurrence.

With its unique fast-slewing and flexible observing scheduling, which makes a monitoring effort cost-effective, its broad-band energy coverage that would allow us to model the observed spectra simultaneously in the $0.3-150 \mathrm{keV}$ energy range, thus testing the prevailing models for accreting neutron stars, and the high sensitivity in the soft X-ray regime, where some of the SFXTs had never been observed, Swift was the most logical choice to monitor the light curves of our sample in order to search for the outburst recurrence predicted by our model.

Our targets (IGR J16479-4514, XTE J1739-302/IGR J17391-3021, IGR J17544-2619, and AX J1841.0-0536/IGR J18410-0535) were selected considering sources which, among several SFXT candidates, are confirmed SFXTs, i.e. they display both a "short" transient (and recurrent) Xray activity and they have been optically identified with supergiant companions (see references in [25]). In particular, XTE J1739-302 and IGR J17544-2619 are generally considered prototypical SFXTs. XTE J1739-302 was the first transient which showed an unusual X-ray behaviour [23], only recently optically associated with a blue supergiant [9]. IGR J16479-4514 has displayed a more frequent X-ray outburst occurrence than other SFXTs [25] and offers an a priori better chance to be caught during an outburst. AX J1841.0-0536, on the other hand, is an interesting source which may offer the opportunity to determine the orbital parameters from the pulsar timing 


\begin{tabular}{lrrrrr}
\hline \hline Name & Campaign Start & $\begin{array}{r}\text { Number of } \\
\text { Observations }\end{array}$ & $\begin{array}{r}\text { Swift/XRT } \\
\text { Exposure }(\mathrm{ks})\end{array}$ & $\begin{array}{r}\text { Outburst } \\
\text { Dates }\end{array}$ & References \\
\hline IGR J16479-4514 & $2007-10-26$ & 58 & 67 & $2008-03-19$ \\
XTE J1739-302 & $2007-10-27$ & 83 & 104 & $2008-04-08$ \\
& & & & $2008-08-13$ \\
IGR J17544-2619 & $2007-10-28$ & 57 & 58 & $2007-11-08$ \\
& & & & $2008-03-31$ \\
AX J1841.0-0536 & $2007-10-26$ & 71 & 77 & none
\end{tabular}

Table 1: Status of the Swift monitoring campaign as of September 10, 2008.

on long time scales.

For these sources we requested 2-3 observations week ${ }^{-1}$ object $^{-1}$, each 1 ks long with Swift/XRT [3] in AUTO mode, to best exploit XRT automatic mode switching [6] in response to changes in the sources' observed count rates. This observing pace would naturally fit in the regular observation scheduling of $\gamma$-ray bursts (GRBs), which are the the main observing targets for Swift. We also planned to propose for further target of opportunity (ToO) observations whenever one of the sources showed interesting activity, (such as indications of an imminent outburst) or underwent an outburst, thus obtaining a finer sampling of the light curves and allowing us to study all phases of the evolution of an outburst.

With this setup, we aimed at fully characterizing the long-term behavior of SFXTs, to determine the properties of their quiescent state (where the accumulation of large observing time is needed to allow a meaningful spectral analysis of this faintest emission), to monitor the onset of the outbursts and to measure the outburst recurrence period(s) and duration.

Up to September 10 2008, we have collected a total of $306 \mathrm{ks}$ distributed as shown in Table 11. The XRT light curves of the campaign are shown in Figure 1. The long-term X-ray emission outside the bright outbursts is described in full in [19], while the first observed outbursts of IGR J16479-4514, IGR J17544-2619, and IGR J17391-3021, are reported on in [13] and [19], respectively. Furthermore, in these Proceedings [22] we report the preliminary results on two more outbursts of IGR J17544-2619 and XTE J1739-302. Here we shall highlight the results on the 2008 March 19 outburst of IGR J16479-4514 and on a Swift GI observation on the 2008 June 16 outburst of IGR J11215-5952.

\section{Rise to the outburst in IGR J16479-4514}

The first outburst that triggered the Swift/BAT since the beginning of the campaign occurred on 2008 March 19 at 22:44:47 UT, and then shortly thereafter at 22:59:59 UT ([13]), when IGR J16479 -4514 reached fluxes above $10^{-9} \mathrm{erg} \mathrm{cm}^{-2} \mathrm{~s}^{-1}$, which translates into a luminosity of about $6 \times 10^{37}$ $\mathrm{erg} \mathrm{s}^{-1}(0.5-100 \mathrm{keV}$; at a distance of $4.9 \mathrm{kpc})$. Swift immediately re-pointed at the target with the narrow-field instruments so that, for the first time, an outburst from a SFXT where a periodicity in the outburst recurrence is unknown could be observed simultaneously in the $0.2-150 \mathrm{keV}$ energy band (Figure 2). We observed a highly variable $\mathrm{X}$-ray emission that spans almost four orders of 

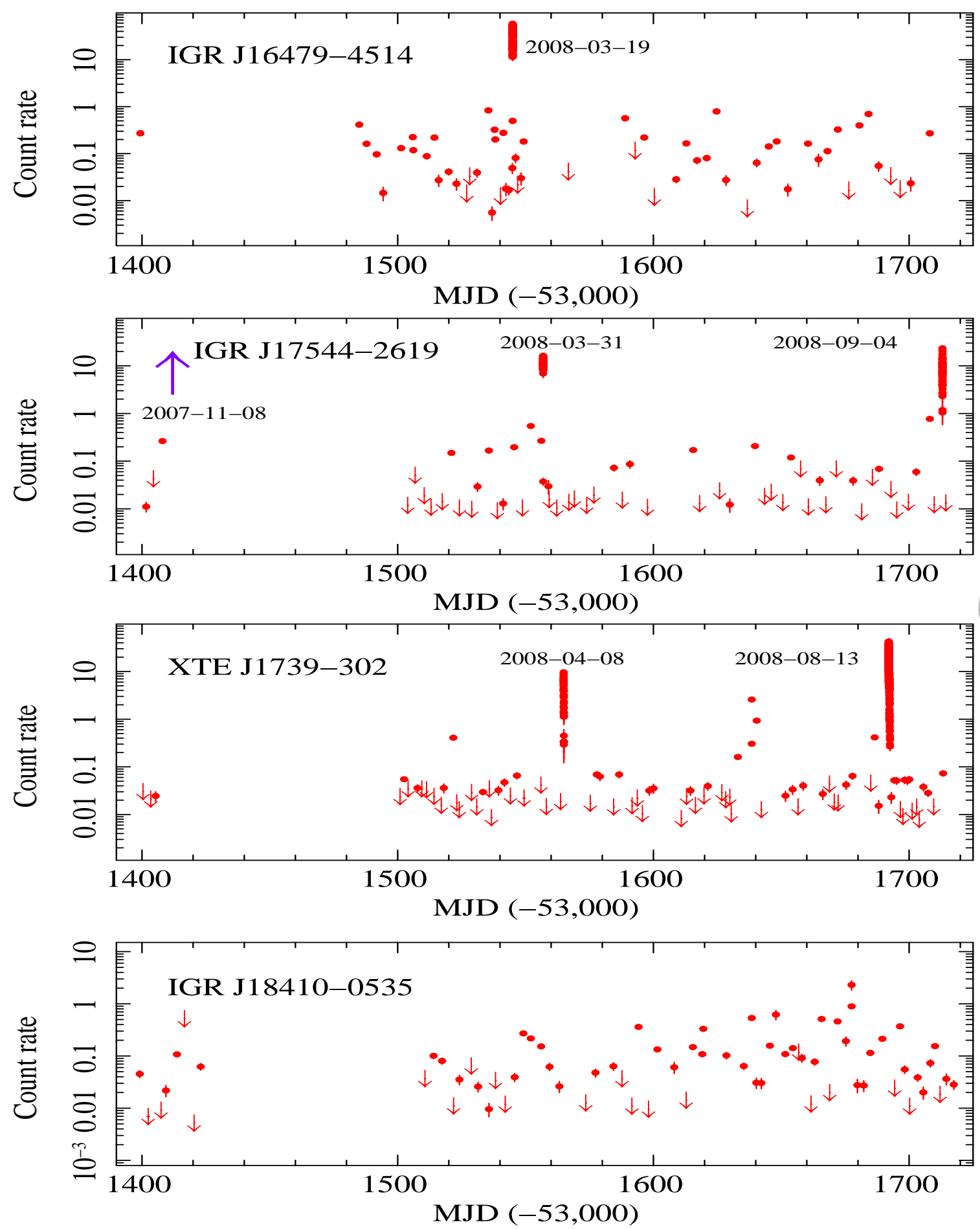

Figure 1: Swift/XRT light curves of our sample of 4 SFXTs in the 0.2-10 keV energy range, between 2007 October 26 and 2008 September 10. The light curves are background subtracted, and corrected for pile-up (when required), PSF losses, and vignetting. In each panel we report the dates of the observed outburst. Downward-pointing arrows are 3- $\sigma$ upper limits, while the upward pointing arrow in the light curve of IGR J17544-2619 marks the 2007-11-08 outburst that XRT could not observe because the source was Sunconstrained. The gap in the data points between November 2007 and January 2008 is due to the sources being Sun-constrained. 


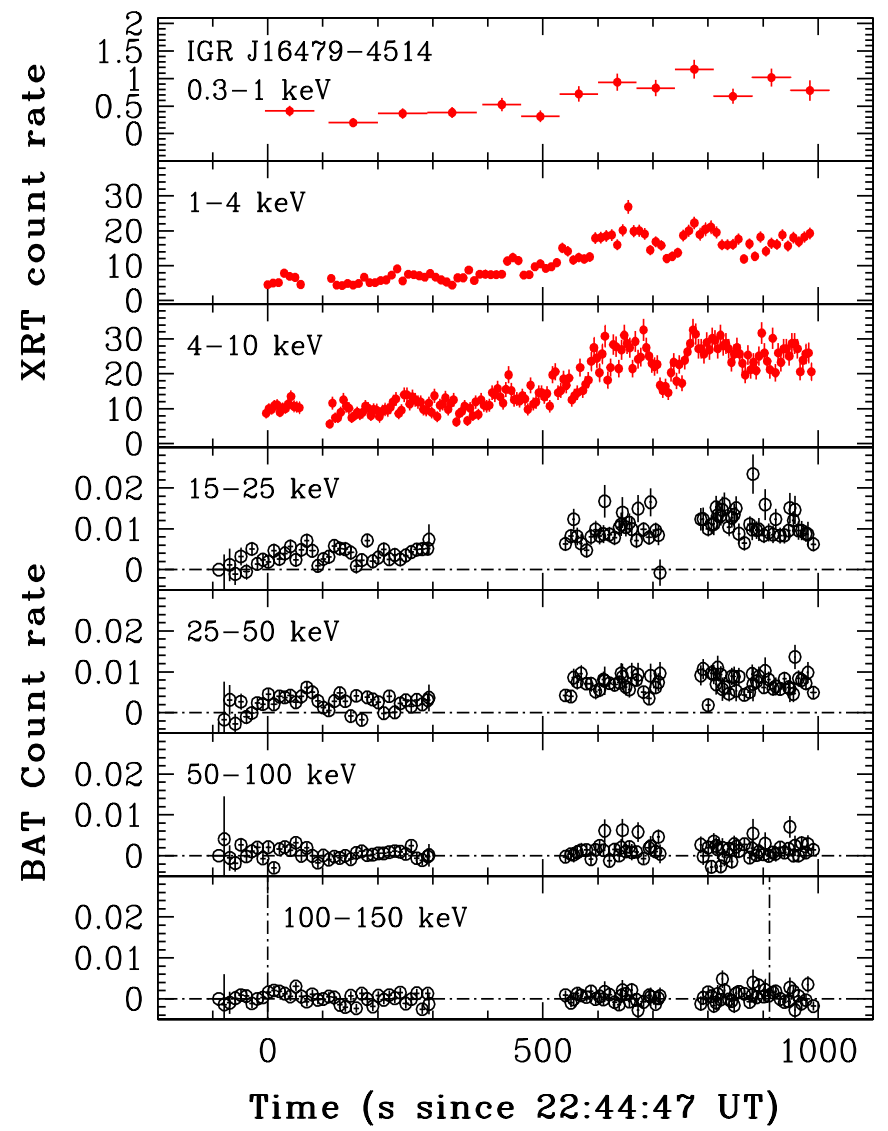

Figure 2: XRT (red filled circles) and BAT (black empty circles) light curves of the 2008 March 19 outburst of IGR J16479-4514 in units of count $\mathrm{s}^{-1}$ and count $\mathrm{s}^{-1}$ detector $^{-1}$, respectively. The vertical dot-dashed lines in the bottom panel mark the two BAT triggers. The XRT points up to $\sim 60 \mathrm{~s}$ after the first BAT trigger were collected as a pointed observation part of our monitoring program. The gaps in the BAT data are caused by BAT event mode time intervals being limited to less than $600 \mathrm{~s}$ to reduce telemetry.

magnitude in count rate during the Swift/XRT observations covering a few days before and after the bright peak. The XRT spectrum in outburst is hard and highly absorbed (the power-law fit resulted in a photon index of $0.98 \pm 0.07$, and in an absorbing column density of $\sim 5 \times 10^{22} \mathrm{~cm}^{-2}$ ). For the first time a simultaneous broad band X-ray spectrum of IGR J16479-4514 (also a first in the SFXT class) was analyzed in the $0.3-100 \mathrm{keV}$ energy range. The source emission is fit well with the spectral models usually applied to the accreting $\mathrm{X}$-ray pulsars: power laws with a high energy cutoff models (Figure 3 ). The resulting parameters are also very similar to those of this kind of X-ray binary sources.

\section{Disentangling the system geometry in IGR J11215-5952}

Presently, IGR J11215-5952 is the first of two SFXTs displaying periodic outbursts (the second one being IGR J18483-0311 which shows outbursts with a period of 18.52 days, [17]). While INTEGRAL and RXTE observations have shown that the outbursts occur with a periodicity of $P=329$ days, thanks to our Swift data we have firmly established that the true outburst period 


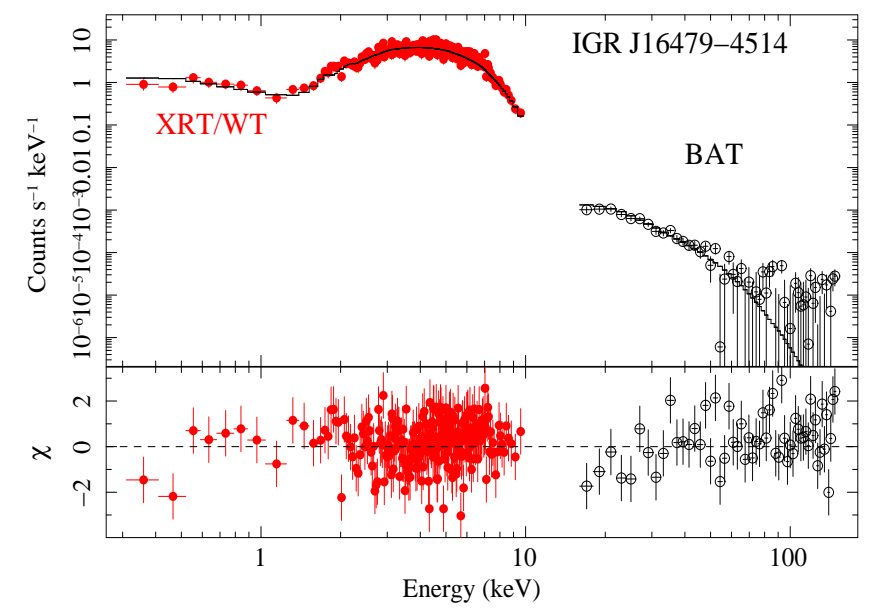

Figure 3: Spectroscopy of the 2008 March 19 outburst of IGR J16479-4514. Top: data from the second BAT observation (black empty circles) and simultaneous XRT/WT data (red filled circles) fit with an absorbed power law with a high energy cutoff. Bottom: the residuals of the fit in units of standard deviations.

is $\sim 165$ days. In fact, we observed this source with Swift several times so that our data sample includes (Figure Ð):

1. 2007 February monitoring, intense 23 -day long campaign intended to study the fifth outburst ("periastron" on February 9, based on a $329 \mathrm{~d}$ period, [11]), when we could study the evolution of the light curve from outburst onset to almost quiescence, and which constitutes a unique data-set for an outburst of a SFXT, thanks to the combination of sensitivity and time coverage.

2. 2007 July (26-day long) monitoring to study the following "apastron" (July 24 , based on $P=329 \mathrm{~d}$, [18]) . We obtained a ToO for $1.5-2.5 \mathrm{ks} \mathrm{day}^{-1}$ from June 5 to July 31 , and 17 ks on-source time. A new, unexpected outburst was observed with spectral and energetic properties fully consistent with the ones observed during the periastron outburst. The mean spectrum of the bright peaks can be fit with an absorbed power law model with a photon index of $1.00_{-0.14}^{+0.16}$ and an absorbing column of $\left(1.04_{-020}^{+0.25}\right) \times 10^{22} \mathrm{~cm}^{-2}$. This outburst reached luminosities of $\sim 10^{36} \mathrm{erg} \mathrm{s}^{-1}(1-10 \mathrm{keV})$.

3. 2008 March $25-27$ observations to study the $P / 4$ (based on $P=329 \mathrm{~d}$, [12]). No emission was observed down to a 3- $\sigma$ upper limit of $9.1 \times 10^{-13} \mathrm{erg} \mathrm{cm}^{-2} \mathrm{~s}^{-1}\left(\Gamma=1\right.$ and $N_{\mathrm{H}}=10^{22}$ $\mathrm{cm}^{-2}$ ), therefore we can exclude that the period is $165 / 2$ days.

4. 2008 June monitoring (a $20 \mathrm{ks}$ GI observation followed by follow-up observations for a total of $12 \mathrm{ks}$, [16]) between the predicted outburst ("apastron", based on $P=329 \mathrm{~d}$, June 16) and July 4.

The characteristics of this latest "apastron" outburst (2008 June 16) are quite similar to those previously observed during the "periastron" outburst of 2007 February 9, both in terms of spectral shape and luminosities reached. The light curve can be modelled with the parameters obtained by [18] for the 2007 February 9 outburst, although some differences can be observed in its shape. The 


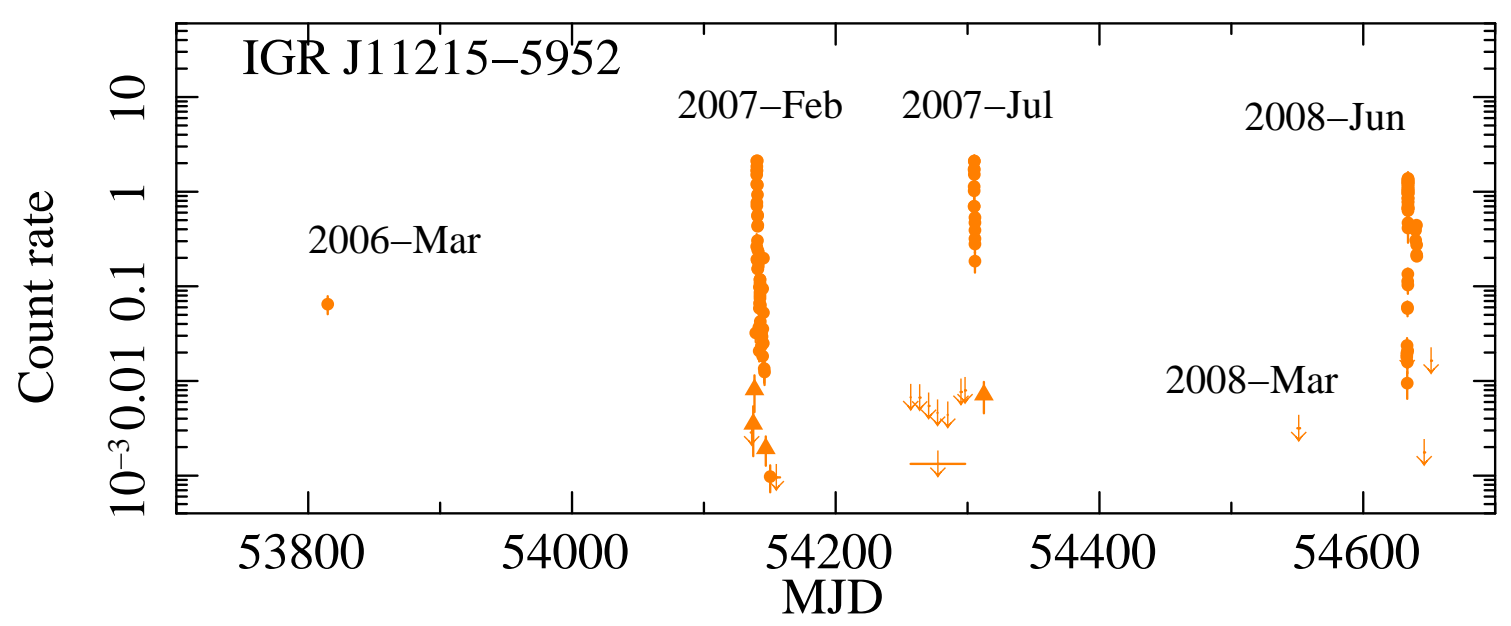

Figure 4: XRT full light curve of IGR J11215-5952 (1-10 keV); the curve is background subtracted, and corrected for pile-up (when required), PSF losses, and vignetting. Downward-pointing arrows are 3- $\sigma$ upper limits.

properties of the rise to this new outburst and the comparison with the previous outbursts allow us to suggest that the true orbital period of IGR J11215-5952 is very likely 164.6 days, and that the orbit is eccentric, with the different outbursts produced at the periastron passage, when the neutron star crosses the inclined equatorial wind from the supergiant companion. Based on the observation performed on 2008 March 25-27, we can exclude that the period is $165 / 2$ days.

\section{Conclusions}

Our almost year-long campaign on this new class of HMXBs has demonstrated how the unique flexibility of Swift, paired with the high sensitivity of its instruments, allowed us to monitor as continuously as possible the onset of the outbursts in these systems. We have obtained multiwavelength observations for a total of 5 outbursts from 3 sources, which we were able to study in great detail through wide-band spectroscopy, and time resolved spectroscopy at soft X-rays. We have shown (also see [22]) that common characteristics to this class of objects are emerging, i.e., outburst lengths well in excess of hours, often with a multiple peaked structure, dynamic range $\sim 3$ orders of magnitude. Finally, periodicities are starting to be found.

\section{Acknowledgments}

We thank the Swift team duty scientists and science planners P.J. Brown, M. Chester, E.A. Hoversten, S. Hunsberger, C. Pagani, J. Racusin, and M.C. Stroh for their dedication and willingness to accomodate our sudden requests in response to outbursts during this long monitoring effort. We also thank the remainder of the Swift XRT and BAT teams, J.A. Nousek and S. Barthelmy in particular, for their invaluable help and support with the planning and execution of the observing strategy. This work was supported in Italy by contracts ASI I/023/05/0 and I/088/06/0, at PSU by NASA contract NAS5-00136. H.A.K. was supported by the Swift project. P.R. thanks INAF-IASF Milano and L.S. INAF-IASF Palermo, for their kind hospitality. Italian researchers acknowledge 
the support of Nature $(455,835-836)$ and thank the Editors for increasing the international awareness of the current critical situation of the Italian Research.

\section{References}

[1] Bamba, A., Yokogawa, J., Ueno, M., et al., 2001, PASJ, 53, 1179

[2] Bozzo, E., Falanga, M., Stella, L., 2008, ApJ, 683, 1031

[3] Hill, J. E., Burrows, D. N., Nousek, J. A., et al., 2005, SSRv, 120, 165

[4] Cusumano, G., Romano, P., Sidoli, L., et al., 2009, MNRAS, in preparation

[5] Gehrels, N., Chincarini, G., Giommi, P., et al., 2004, ApJ, 611, 1005

[6] Hill, J. E., Burrows, D. N., Nousek, J. A., et al., 2004, SPIE, 5165, 217

[7] in't Zand, J.J.M., 2005, $A \& A$, 441, L1

[8] Krimm, H. A., Barthelmy, S. D., Barbier, L., et al., 2007, The Astronomer's Telegram, 1265

[9] Negueruela, I., Smith, D.M., Reig, P., et al. 2006, in ESA Special Publication, ed. A. Wilson, Vol. 604, 165

[10] Negueruela, I., Torrejon, J.M., Reig, P., et al., 2008, AIPC, 1010, 252

[11] Romano, P., Sidoli, L., Mangano, V. et al., 2007, A\&A, 469, L5

[12] Romano, P., Mangano, V., Mereghetti, S., et al., 2007, The Astronomer's Telegram, 1151

[13] Romano, P., Sidoli, L., Mangano, V., et al., 2008, ApJ, 680, L137 [Paper II]

[14] Romano, P., Guidorzi, C., Sidoli, L., et al., 2008, The Astronomer's Telegram, 1659

[15] Romano, P., Cusumano, G., Sidoli, L., et al., 2008, The Astronomer's Telegram, 1697

[16] Romano, P., Sidoli, L., Cusumano, G., et al., 2009, ApJ, in press

[17] Sguera, V., Hill, A. B., Bird, A. J., et al., 2007, A\&A, 467, 249

[18] Sidoli, L., Romano, P., Mereghetti, S., et al., 2007, A\&A, 476, 1307

[19] Sidoli, L., Romano, P., Mangano, V., et al., 2008, ApJ, 687, 1230 [Paper I]

[20] Sidoli, L., Romano, P., Mangano, V., et al., 2009, ApJ, 690, 120 [Paper III]

[21] Sidoli, L., 2008, Proc. of the COSPAR Assembly 2008, 37, 2892, [arXiv:0809.3157]

[22] Sidoli, L., Romano, P., Cusumano, G., et al., 2009, in proceedings of 7th INTEGRAL Workshop, PoS (Integral08) 084, [arXiv:0810.5446]

[23] Smith, D. M., Main, D., Marshall, F., et al., 1998, ApJL, 501, 181

[24] Swank, J.H., Smith, D.M., Markwardt, C.B., 2007, The Astronomer's Telegram, 999

[25] Walter, R., \& Zurita Heras, J., 2007, A\&A, 476, 335 